\title{
A Location-based Interface Approach to Developing a Systematic Event Analysis Tool
}

\author{
C. Madden ${ }^{\mathrm{a}}$ \\ ${ }^{a}$ Defence Science and Technology Group \\ Email: Christopher.Madden@dst.defence.gov.au
}

\begin{abstract}
Forensic investigations of events involving significant damage or a loss of life require collecting and collating a vast amount of systematic data about the event to determine what happened. This can include maps of the area, plan drawings of any platforms involved, imagery, videos and witness accounts, repair histories along with a significant amount of other data that might be available. Advances in computing and multimedia technology provide new approaches for investigators to collate this data. They can also allow for other stakeholders to better interact with and contextualize the data to improve their understanding of what happened during the event.
\end{abstract}

This paper outlines techniques used to develop a location-based interface prototype for an interactive tool that allows users to intuitively explore and analyse data about an event. This prototype can easily add data in various forms, including the timeline of what occurred before, during and after the actual event, with the data positioned manually in the current prototype. The tool collates the data onto a map, plan drawing or (in future versions) a 3D model based upon the location where the data was captured. This allows users to move through the data spatially, keeping an intuitive sense of their relevant locations and eliminating the need to jump to different folders or data categories as they explore the data. With accurate recording of the locations where the data was captured, automated processes could collate the data, allowing a broader range of users to more quickly understand how the data fits together to indicate what happened during the event. Once the data analysis is finished, the tool can also visually demonstrate the findings of the investigation. The speed of data collation and investigation can make it useful for examining the cause of even minor events, which have less data collection than major incidents.

This paper provides an example of the prototype tool using data obtained from experiments on the ex-HMAS Derwent, which was used to investigate blast damage on a naval vessel. The paper shows how images, video, audio and written notes can be connected to the plan drawings of the ship based upon the location they were captured. It also demonstrates how overlays can be used to add additional information. Additional imagery demonstrates how this data can also be attached to 3D models of the platform where they are available. Such models could be used as part of Augmented or Virtual Reality interfaces. This would allow users to see data overlaid on top of a 3D model or scan of the platform or to create scenes where a user could potentially walk or fly through a virtual reconstruction of the event.

A similar type of tool to that used for event analysis could be used to collate information for a range of other uses. For example, this could be used to store the construction and maintenance history of individual platforms, whether in the land, sea or air domains. This tool could include readily accessible information of minor differences, such as electrical wiring or when and where components were last tested or painted. This information could be used to better inform and tailor maintenance and repair regimes, especially when issues arise while a ship is at sea, as the information could be deployed on-board as a readily accessible application. Appropriate authentication techniques must be included to ensure the security of the tool and the data, and that the user has access to the appropriate data. As such tools can run on low power devices connected to the data storage for the investigation, they could constantly update and provide the latest information, even during a maintenance cycle or during field operations.

Keywords: Event analysis, data collection, multimedia interface, location-based interface 


\section{INTRODUCTION}

Any event that causes significant damage or a loss of life should be investigated to identify the causes and mitigate them to reduce the chance of that incident reoccurring. Conducting such an investigation is a lengthy process of systematically collecting a wide range of data about not only what happened during the incident, but also the situation leading up to the incident and what occurred afterwards. Whilst photographs or videos form a proportion of the data about an incident, various other pieces of data are crucial for contextualizing that information. This ranges from maps of the area, plan drawings of the structures or platforms involved, to details about electrical wiring, fuel loads, insulation or other data relevant to the incident that occurred. The collection of this data can take significant time and collating it into a useful data repository for investigators and other stakeholders is a challenging task. Advances in user interface design techniques and tools allow for new methods to obtain and access this data rather than the traditional folder based filing approach. Where minor events occur, which do not qualify as a significant incident, data is likely to be captured, but is often not analysed due to the challenge of exploring that data efficiently and effectively.

The final product of an incident investigation is typically a report for the stakeholders about the cause of the incident. Therefore the data included in the report is selected based upon its suitability for the format as well as its importance to the findings, which may lead to the conversion of information into a report ready format. A combination of data conversion and the limited space in the report means that much of the data is not included in the final report. Given the volume of data that may be captured, it may also be quite difficult for many stakeholders who are not intimately involved in the investigation to effectively explore the stored data to gain additional insights into the event that may be important but not explicitly reported. For instance audio is often transcribed to a written account, which may ignore some of the informative background noise, or at best only includes key sounds. This may lose some of the context for the reader of the report when compared to listening to the audio recording. Whilst this may not be critical to the exact cause of the incident, it could be of use when considering overall design changes to a structure. Without an intuitive interface to the data, such as the prototype proposed in this paper, exploration of the data can be difficult.

Advances in computing power and software design allow for a wide range of more intuitive User Interface (UI) designs to be developed to have more natural and familiar interfaces, such as using a location-based rather than folder based interfaces (Haklay and Zafiri, 2008). Depending upon the actual event being investigated these could include maps of the area, plan drawings of any buildings or platforms involved, or could even include 3D models. By linking the access of specific data to physical locations, it creates a more natural understanding of the context around the data being presented, whether it is an image, video, audio or even text. Using multimedia techniques, such as metadata collection and image overlays, the interface can also be designed so that it is generated quickly and allows a user to see the broad overview of the available data, or to focus in on specific areas or details. This paper uses data obtained from the Ship Survivability Enhancement Program (SSEP) experiments on ex-HMAS Derwent to demonstrate an example prototype of this interface. More details on the SSEP experimental program are provided in section 4.

Advances in the design of smart data collection devices has led to not only an increasing amount and resolution of data, but also the collection of metadata as the data was captured. This metadata can include details such as the devices location and orientation, which whilst useful for manual data collation, can also be processed to automatically collate the data as it is captured. It is important to consider that the data is potentially very sensitive, so a secure network connection and authentication process should be used. Through the combination of advanced location-based user interfaces, smart data capture devices and a secure network connection, users can more quickly add to or explore the data that is systematically collected about an event, whether it is a serious international incident, or of a smaller nature.

\section{TYPES OF MULTIMEDIA DATA COLLECTED FOR SYSTEMATIC EVENT ANALYSIS}

Conducting an investigation into an event requires the systematic collection of a large amount of data. This can take a long time for significant incidents, but is often not conducted for minor events. This is partly because of time to collect all the data, but also because methods for efficiently collating and interacting with the data have not been fully developed. The development of smarter data capture devices makes it possible to automate much of the data collation process; however data must be processed and organised based upon their data type. The key data types used to develop the interface prototype are described in this section and include Photography, Videos, Audio, Text-based information, and 3D models, though some other data types may also be available. The important metadata for each of these data types includes the location, orientation and time that the data was captured. For a small set of data the location and orientation may not be relevant, such as recording witness statements, so this data can be grouped together on the interface. The examples provided here are based upon the SSEP experiments on ex-HMAS Derwent, which are described in section 4. 


\subsection{Imagery}

The predominant data collected for an investigation about an event are photographs. This occurs because photographs can be quickly and cheaply captured and reviewed. They can show a range of features about the area being viewed and can be quickly compared to previous images about that area to highlight changes that occurred during the event. A range of other imagery is also important to an investigation, such as maps of the area, plan drawings of any structures or platforms involved and diagrams of key information such as insulation or fuel loads for fires. These are often presented as an image or image overlay as it is a common format used in a report. This imagery tends to have a form that demonstrates the understanding of its state at that time, such as the insulation installed at that time, or fuel loads in that area when the event started.

The volume of photography taken before, during and after an event depends upon the event, though imagery usually forms the largest number of data points. This is because photographs are taken from different angles to show multiple perspectives of the damage. Metadata on both the location the imagery was taken and the orientation of the imaging device should be captured. The accuracy of this metadata can be very important, especially when placing the imagery in a 3D environment, as an inaccurate location or misalignment of the imagery may make a user mistrust the imagery. For planned events, imagery is captured prior to the event to compare and highlight the damage that occurred during or after the event. To differentiate between such data, the time of data capture is crucial. Where an event is unexpected, limited imagery may be available to understand the original condition of an area. Figure 1 shows the ex-HMAS Derwent plan drawings, with Figure 2 showing examples of imagery captured during and post the SSEP fire experiments.

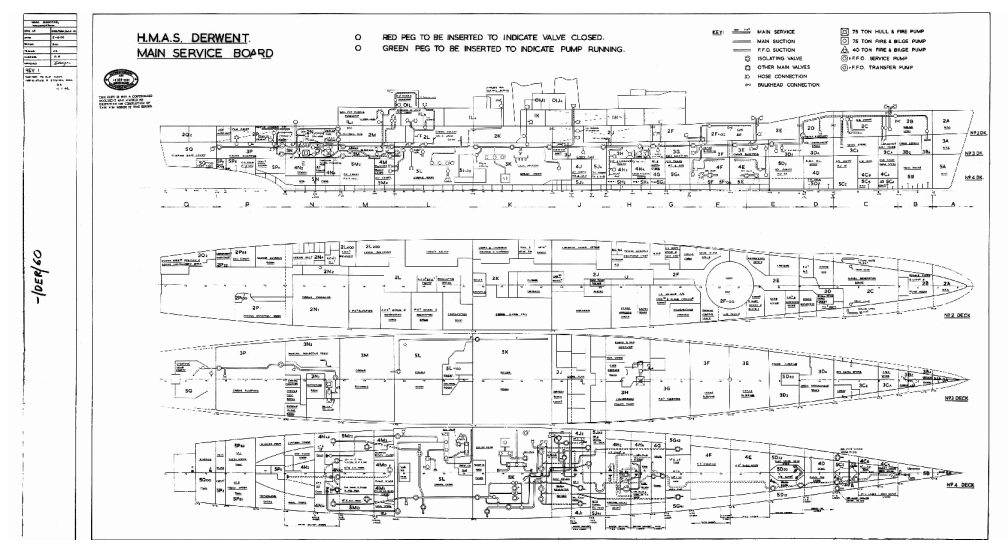

Figure 1. ex-HMAS Derwent Plan Drawing

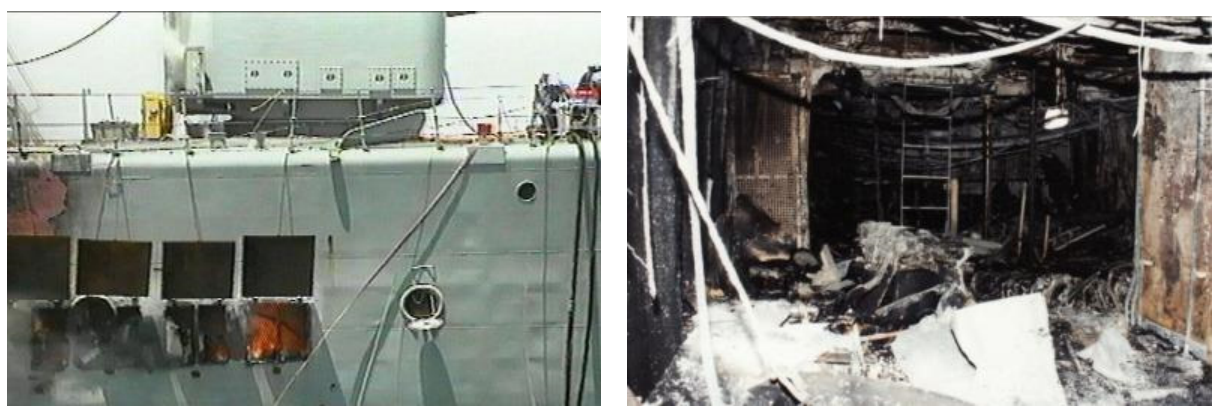

Figure 2. Example fire images both during and post a fire experiment on ex-HMAS Derwent

\subsection{Videos}

Video sequences can also be easily captured using a range of modern devices and even smartphones. They differ from imagery as they are a sequence of images and potentially corresponding audio, which show the changes over the duration of the video sequence. Videos of the actual event occurring can be valuable objective sources of information about what actually occurred during the event, though the location and orientation of the video camera will determine if it actually captures the key details. Depending upon the size, speed and how unexpected an event is, it is difficult to capture all the important information about the event, especially if only a handheld single camera is available at the time. Ideally multiple cameras at various viewpoints could capture the entire event; however this is only likely to occur where the event is a preplanned experiment or if it occurs in an area under high surveillance. 
The location, orientation and timing of the video data that is capturing serious damage to a structure will be very important; however for other videos, such as witness statements, only the timing may be relevant. An automated data collation tool is likely to have some difficulty determining this difference, though a simple switch allowing a user to designate a video or other data as location independent could be easily implemented. This would allow for such videos to be collected at a predetermined area of the interface. Figure 3 shows the prototype interface that was developed with an embedded video being displayed.

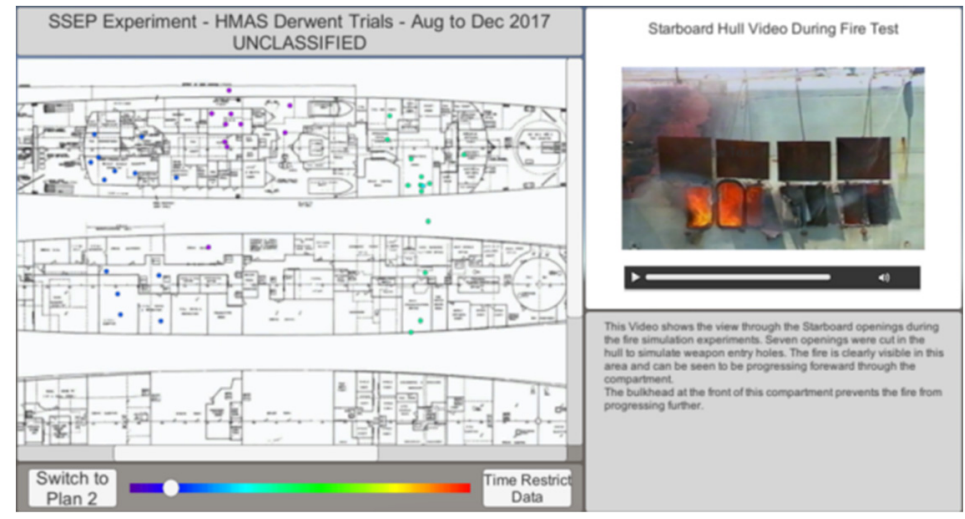

Figure 3. Example of video data located on the prototype interface for an ex-HMAS Derwent experiment

\subsection{Audio}

Noises during an event can be useful to analyse the order in which actions occurred, but are often not collected using directional microphones, so only the location and timing is available. Incident reports usually include a written transcript of important audio information as it is difficult to embed audio in a printed report. Interested stakeholders therefore have to access the data store to hear the original recording. In digital tools, such audio can be included as a data point based upon its location data, or the location of the data it is associated with, such as for an audio annotation for imagery. As with other data types, location independent data could be manually positioned to cluster with other related data, such as with other witness accounts.

\subsection{Text}

Text based information can be used in a variety of ways from short annotations about other data sources to written transcripts of events to supporting reports. Depending upon the type of text included, they will often be linked to already existing data and should most likely be displayed along with that data as an appropriate text box. Where the textual data consists of a full supplementary report, it should be located with the information that it is supporting, or potentially in a general documents area.

\subsection{D Models}

With the increasing capability of computers, it is now possible for most computers to display and interact with 3D models of an area or structure. For all but the very high end computers, a 'low polygon' model is required. This maintains the overall shape of the structure, but simplifies it compared to a highly detailed Computer Aided Design (CAD) model. This allows a user to see and interact with the essential shape of the 3D object, but not see the detail of every nut and bolt. This allows for a comparison to be made between the platform structure and the imagery obtained, identifying any changes that may have occurred. If the metadata of imagery or audio data points is accurate, then it can be automatically placed into the scene to facilitate any comparisons, but misalignment of the imagery can cause it to be perceived as being less accurate by the user. Figure 4 shows a 3D model of ex-HMAS Derwent with the hull transparent to show internal compartments.

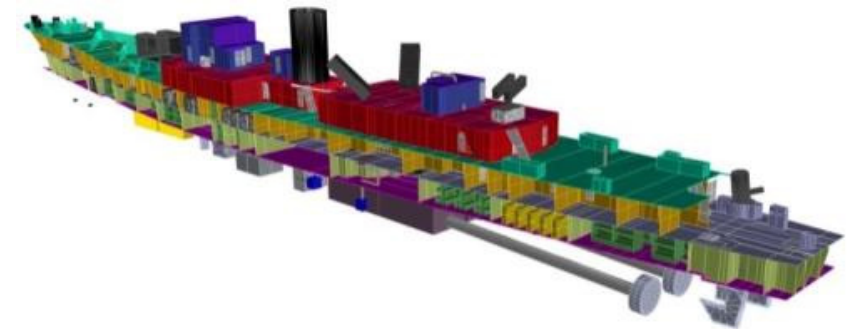

Figure 4. Example of 3D model of ex-HMAS Derwent with the hull invisible to show all the compartments 


\subsection{Other data}

Whilst much of the data collected about an event fits into the above categories, some does not. Such data can often be converted into one of the previously mentioned data types. For instance information about fire insulation thicknesses can be converted to a graphical representation that might be overlaid on a set of plan drawings. Data such as object deformations or temperature changes might be better represented as an image overlay as shown in Figure 5. Information like 3D scans and point clouds could be displayed similar to 3D model information. The locations and movements of people and objects during an event could be represented as a video overlay or lines indicating the direction of movement. Some information is more ambiguous such as weather information as ambient temperature could be a simple textbox, whilst rainfall and cloud cover might be video overlays. The flexible nature of the computer programs provide the user with a range of options for incorporating such data into the overall tool depending upon the best way for it to be displayed.

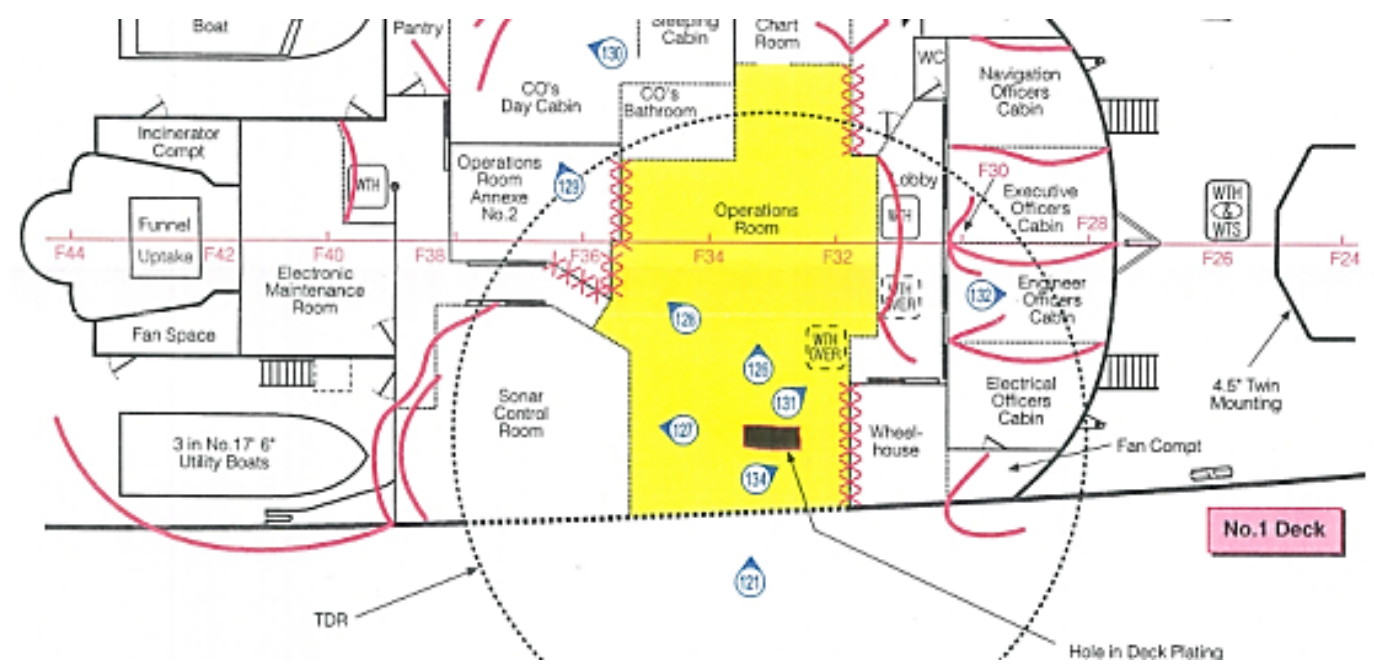

Figure 5. Example of a deformation damage overlay for a warhead detonation experiment on ex-HMAS Derwent

\section{CREATING A LOCATION-BASED INTERFACE FOR THE MULTIMEDIA DATA}

Creating a data investigation tool that makes it easy to collate and interface with the data collected can not only increase the speed of an investigation, but also make smaller scale investigations plausible. Two key aspects which make an interface easy to use are having an obvious logical structure and being familiar to the user (Blackler et. al. 2006). Given that the data collected for an investigation is generally based upon changes or effects at key locations around the structure or area, a location-based interface is an obvious logical structure. This structure for an interface has become widely used for a variety of Geographic Information Services (GIS) (Jiang and Yao, 2006). This structure plots data points as icons based upon their location, which remain in place as a user scrolls around or zooms in and out to find features or data of interest.

The location-based interface proposed for a particular investigation would depend upon the event, but could be based around an area map, a set of plan drawings, or 3D models. For some applications, a combination of two or even all of these modalities could be used. The map style interface would be most appropriate where the event occurs in parts spread over an area, or for demonstrating the spread of locations of a debris field. GPS coordinates can easily be converted to identify the appropriate location on a map. A plan drawing is most appropriate where an investigation is focussed on a particular structure or platform, such as a building or a ship. GPS may not penetrate inside a structure though, so reliable locations may often require a different location system, such as beacon localisation or the use of Wi-Fi fields (Deak et. al. 2012). Where 3D models are available, they can be used as a reference structure for the investigation data once a translation from world coordinates to the model coordinates is determined. Even more care needs to be taken when placing imagery onto a $3 \mathrm{D}$ model as even small errors in the alignment of the location or orientation of imagery to the model can make the imagery look inaccurate. Whilst stakeholders may understand the reasons for this misalignment, it could make them unnecessarily question the reliability of that particular imagery. Where multiple interfaces are used, a button can be included to switch between the available views.

The location-based nature of the interface allows a user to easily examine imagery clustered around a single location; however the timing of the data is also of significant importance. Whilst a variety of options are available, the current implementation of the data interface has used colour coded icons to represent the timing of when the data was captured, and a slider so users can control the data shown based upon when it was 
captured. This adaptation allows for an investigator to focus down on data obtained at a specific location and time, such as in the lead up to the event or during the event itself as shown in Figure 6 below.

\section{HMAS DERWENT EXAMPLE}

As part of the Ship Survivability Enhancement Program (SSEP), a series of 'live-fire' tests were conducted aboard the former HMAS Derwent from the $1^{\text {st }}$ of August to the $2^{\text {nd }}$ of December 1994 in Cockburn Sound, Western Australia. These tests included the effects of warheads, smoke, fire, and conventional weapons amongst other effects (Walsh et.al. 1996), with the results being used to evaluate and improve the survivability of Royal Australian Navy warships. As this trial occurred over 20 years ago, the cost of collecting and storing large amounts of data was significantly greater than it is currently. Even so data collection included over 20 scenes were recorded with cameras that have a frame rate of up to 5200 frames per second, various pressure transducers and accelerometers as well as photographic evidence and human annotated notes. A prototype of the interface developed for the ex-HMAS Derwent data shown is in Figure 6, with the data manually placed onto the plan drawings and buttons linked to the experimental data.

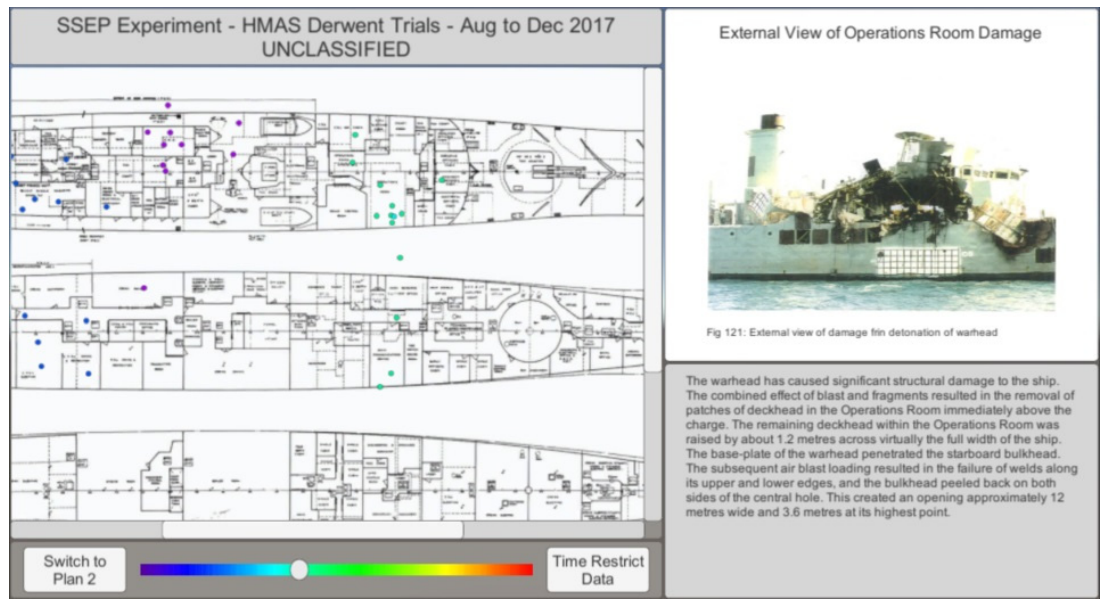

Figure 6. Example interface into SSEP trial on the HMAS Derwent

If the trial was repeated today, a much wider array of cheap time synchronised sensors would be used to create data so that investigators would have not only a significant amount of higher resolution video, but also significantly more real-time data including, but not limited to, temperature, visibility and smoke toxicity in affected compartments, structural deformation, and equipment or power malfunctions. This volume of data would be physically stored from the sensor network onto a large set of hard disks for transfer back to a secure network after the trial was concluded. As the experiments were conducted over an extended time period, this could also be used to distinguish between the data generated for each experiment. By zooming in to the desired location and adjusting the time period to the desired event, the locations of the data points become clearer. Figure 7 below focusses on a warhead experiment that caused significant damage to decks 1 and 2 . The time restriction function has also been activated so only data points collected at this time are shown.

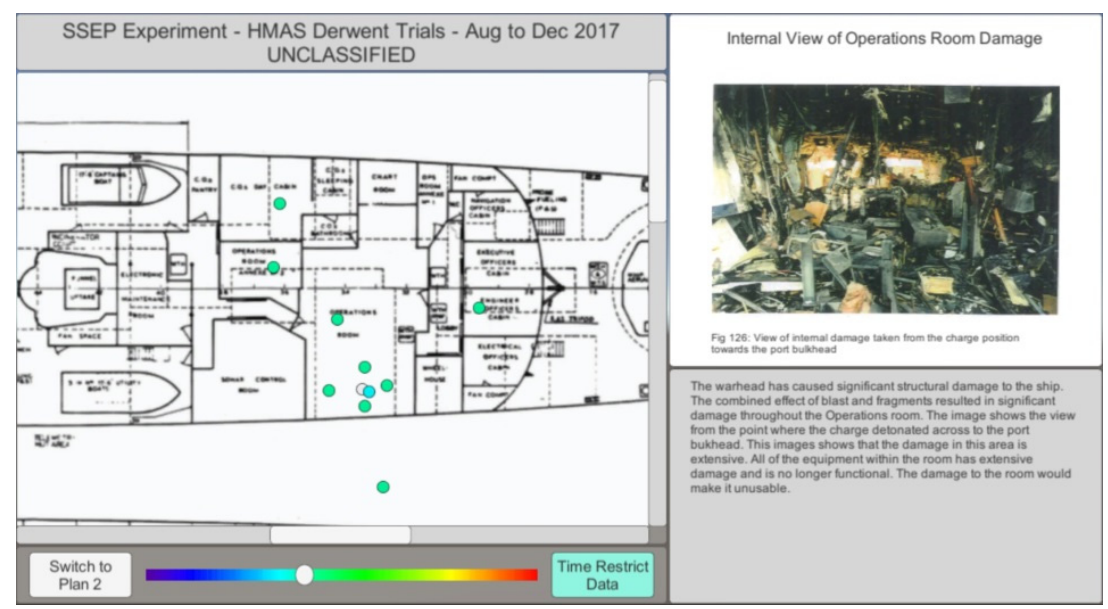

Figure 7. Zoomed view showing decks 1 and 2 with test locations and resulting image 


\section{DISCUSSION OF OTHER USES}

This paper has explored the development and use of a location-based data interface for event investigations; however a similar style of technology could be used for a range of other applications and adapted for other interface technologies. An example would be for the collation of all the data about a particular structure, such as a building, a compound or a military platform. The combination of metadata about the location and time that data is collected would allow for automatic collation of the data to the relevant areas from the initial design, any changes made during construction, repairs and modifications during its operation, through to its decommissioning. The electronic nature of the data store and interface allow for it to be stored even on a mobile platform, though it should be encrypted and protected using appropriate authentication techniques. This would allow for mechanics and engineers to have the most accurate information possible for decision making, whether in the field or maintenance back at base. Where secure communications are available, they could also be used to ensure that a central storage of the information is also kept up to date.

When the location-based data is attached to a 3D Model, it would also allow for Augmented Reality (AR) or Virtual Reality (VR) headsets to be used to explore the data. Using an AR headset the display is see through, allowing the user to see the equipment whilst overlaying the contextual data (Manuri and Sanna 2016). This allows for the contextual display of information for a range of tasks, such as accessing a certain part, or when it was last repaired or replaced. The VR headset differs as it completely replaces a person's view with a computer generated world. Whilst this has been proposed for a range of training tasks, it can also be used to explore the data collated around a virtual model, such as a virtual walk-through. If enough detailed data was available, this could potentially allow for a virtual walk-though as if the event was actually occurring.

\section{CONCLUSION}

This paper has presented a technique for developing a location-based interface for the collation and exploration of data collected as part of an event investigation. It has explained how depending upon the event and the information available, that interface could be based upon a map of the area, plan drawings of the structure or platform, 3D models, or a combination of these. The prototype built around the ex-HMAS Derwent experiments demonstrate how the interface can be used to show locations where information is available and how an investigator can focus on important subsets of the data to examine key features without having to move back and forth through computer folder structures or multiple pages of written report. This ease of interaction with the available data and the ability to automatically collate the data as it is captured could not only create time savings for investigations of incidents with serious damage or a loss of life, but would also allow minor events to be examined using a more thorough and systematic approach.

Plotting data onto a location-based interface has been used in a range of commercial map-based applications, especially for mobile devices. When care is taken to ensure the security of the network between data capture, storage and display devices, such a system can be used for even sensitive commercial or military applications. For example the full maintenance log for a vessel can be carried by mechanics allowing all of them to know all the available knowledge about every part, such as last inspection, last repair, when it was last painted and could even provide instructions on exactly how to identify signs of wear or replace it. An interface similar to the prototype shown has the potential to save time on a range of tasks by providing information quickly and intuitively with potentially more details than traditional paper reports and logs.

\section{REFERENCES}

Nuriminen, A., Jarvi, J. and Lehtonen, M. (2014). A mixed Reality Interface for Real Time Tracked Public Transportation, In $10^{\text {th }}$ ITS European Congress, Helsinki.

Walsh, B.E., Burman, N.M. and Buckland, M.E. (1996). Ship Survivability Enhancement Program (SSEP) Manual of Conventional Weapons Data, DSTO-GD-0105, Defence Science and Technology Organisation.

Manuri, F, and Sanna, A. (2016). A Survey on Applications of Augmented Reality. ACSIJ Advances in Computer Science: an International Journal, 5, (1).

Deak, G., Curran, K. and Condell, J. (2012). A survey of active and passive indoor localisation systems. In Computer Communications, 35(16), 1939-1954

Haklay, M., \& Zafiri, A. (2008). Usability engineering for GIS: learning from a screenshot. The Cartographic Journal, 45(2), 87-97.

Jiang B. and Yao, X. (2006). Location-based services and GIS in perspective, Computers, Environment and Urban Systems, 30(6), 712-725.

Blackler, A.L., Popovic, V. and Mahar, D.P. (2006). Towards a Design Methodology for Applying Intuitive Interaction. In WonderGround: 2006 Design Research Society International Conference, Lisbon. 